An Introduction to

Differential Geometry and Topology in Mathematical Physics 
This page is intentionally left blank 


\section{An Introduction to}

Differential Geometry and Topology in Mathematical Physics

\section{Wang Rong \& Chen Yue}

\section{Zhejiang University, P R China}




\section{Published by}

World Scientific Publishing Co. Pte. Ltd.

P O Box 128, Farrer Road, Singapore 912805

USA office: Suite 1B, 1060 Main Street, River Edge, NJ 07661

UK office: 57 Sheiton Street, Covent Garden, London WC2H 9HE

\section{British Library Cataloguing-in-Publication Data}

A catalogue record for this book is available from the British Library.

\section{AN INTRODUCTION TO DIFFERENTIAL GEOMETRY AND TOPOLOGY IN MATHEMATICAL PHYSICS}

Copyright $\odot 1998$ by World Scientific Publishing Co. Pte. Ltd.

All rights reserved. This book, or parts thereof, may not be reproduced in any form or by any means, electronic or mechanical, including photocopying, recording or any information storage and retrieval system now known or to be invented, without written permission from the Publisher.

For photocopying of material in this volume, please pay a copying fee through the Copyright Clearance Center, Inc., 222 Rosewood Drive, Danvers, MA 01923, USA. In this case permission to photocopy is not required from the publisher.

ISBN 981-02-3559-3

This book is printed on acid-free paper.

Printed in Singapore 


\section{Acknowledgments}

I wish to express my gratitude to Professors D. Y. Gan, B. Y. Hou, and C. L. Shen for several very helpful conversations and suggestions, and to Professor Yue Chen for preparing the English version of my manuscripts.

WANG, RONG 


\section{Preface}

The purpose of writing this book is to give an outline of the classical results and the most recent developments of differential geometry and topology, especially those important developments which are closely related to the new discoveries in the field of theoretical physics. The contents of this book consist of three parts:

In Part I, we introduce the fundamental knowledge of differential manifolds, including exterior differential, tangent space, cotangent space and their transformation properties. Differential operation of tensors on manifolds, Riemann manifold together with its conformal transformation, and complex manifolds are also introduced.

In Part II, the global topological properties of differential manifolds are discussed in details, including the theories of homotopy, homology, de Rham cohomology, fibre bundles and their characteristic classes which were first introduced by S. S. Chern.

In Part III, we emphasize the index theorem and the properties of simply connected 4-manifolds. Atiyah-Singer's index theorem and its applications, such as the derivation of the dimension of instantons' moduli space, are demonstrated. This part also provides an important theorem of Donaldson on intersection forms of 4manifolds, followed by a very exciting conclusion drawn by $\mathrm{M}$. H. Freedman and C. Taubes: "There is an uncountable family of open subsets of $R_{s}^{4}$ which are all homeomorphic to $R^{4}$, but no two are diffeomorphic." This is a very peculiar phenomenon not occurring in any $R^{n}$ with $n \neq 4$, and it happens that the dimension of our universe is $n=4$.

WANG, RONG 


\section{Contents}

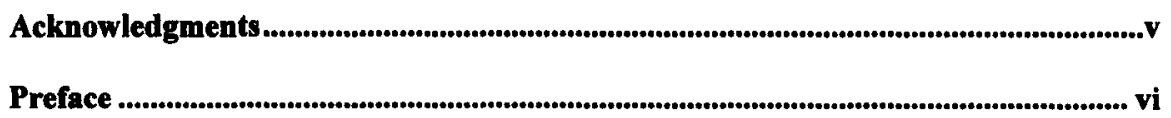

\section{Part I. Differential Manifolds}

Chapter 1. Preliminary Knowledge and Definitions.........................................................1

1.1 What is a Manifold.......................................................................................

1.2 Introducing Coordinates and Differential Structures into Manifolds ......................2

1.3 Tangent Spaces and Cotangent Spaces ...................................................................

1.4 Differential Forms and Exterior Differentials....................................................10

1.5 Orientation of Manifolds and Integration of Differential Forms ...........................13

Chapter 2. Properties and Operations of Tangent Vectors and Cotangent

Vectors ..............................................................................................................................................18

2.1 Mapping Transformation of Tangent Vector Fields and Cotangent Vector

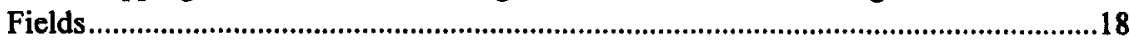

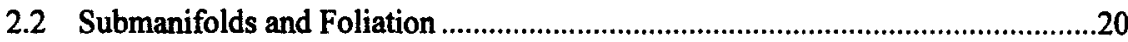

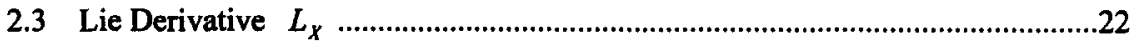

2.4 Interior Product $i_{X}$ and Three Cartan Formulas ..............................................24

2.5 Group Spaces of Lie Groups .....................................................................28

2.6 Invariant Tangent and Cotangent Fields on a Lie Group Manifold M ..................30

Chapter 3. Curvature Tensors, Torsion Tensors, Covariant Differentials and Adjoint Exterior Differentials ..................................................................................................34

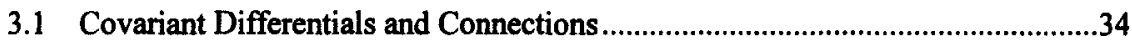

3.2 Curvature, Torsion, and Translation of Vectors on a Manifold ............................37

3.3 Structural Equations and Integrability Conditions of Curvature Tensors and

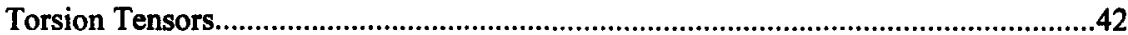

3.4 Hodge * and Adjoint Exterior Differentials.........................................................44

Chapter 4. Riemannian Geometry ........................................................................................50

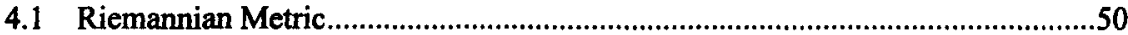

4.2 Levi-Civita Parallel Transport, Riemannian Connection and Curvature Tensor..54

4.3 Two Interesting Examples: ....................................................................................58 


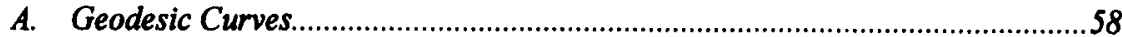

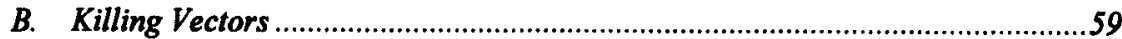

4.4 Vierbeins on an $n$-Dimensional Riemannian Manifold ...................................61

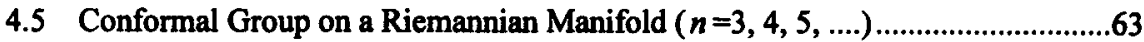

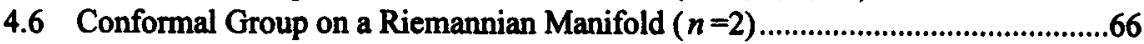

Chapter 5. Complex Manifold....................................................................................................668

5.1 Complex Manifold and its Features ..................................................................68

5.2 Complex Structures and Almost Complex Manifolds on Vector Spaces ..............69

5.3 Almost Hermitian Manifold, Hermitian Manifold and its Connections ................73

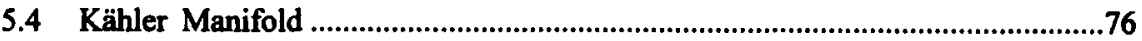

\section{Part II. Global Topological Properties}

Chapter 6. Homotopy Equivalence and Homotopy Groups of Manifolds...............84

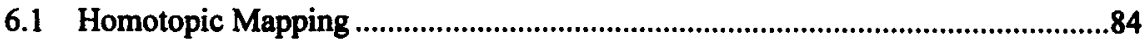

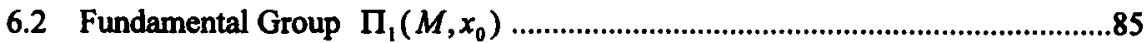

6.3 Homomorphic Sequence and the Structure of Homotopy Groups.........................89

6.4 Higher Order Homotopy Group ...............................................................................94

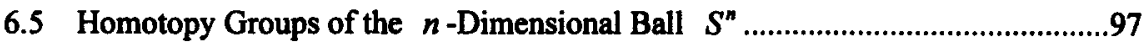

Chapter 7. Homology and de Rham Cohomology .........................................................98

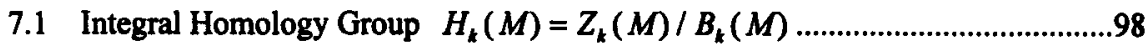

7.2 Universal Coefficient Theorem of Homology Groups........................................104

7.3 Introducing Dual Chain Group and Cohomology Group through Dual

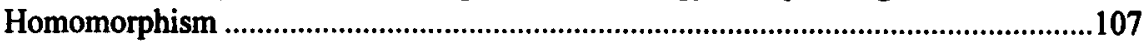

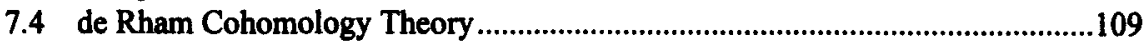

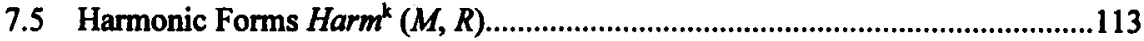

Chapter 8. Fibre Bundles and their Topological Structures .......................................115

8.1 What are Fibre Bundles...............................................................................115

8.2 Fibre Bundles and Cross Sections................................................................118

8.3 Some Representative Fibre Bundles ............................................................119

8.4 A Few Definitions, Principles and Examples of Fibre Bundles ..........................124

8.5 Universal Bundle and Classifying Space ............................................................126

Chapter 9. Connections and Curvatures on Fibre Bundles .........................................130

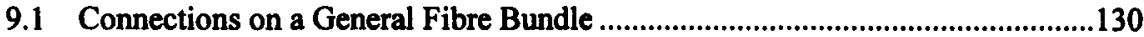

9.2 Remarks on Curvature of Vector Bundles ......................................................134

9.3 Connections on Principal Bundles ...............................................................136

9.4 Connections on Adjoint Vector Bundles ………..............................................143

Chapter 10. Characteristic Classes of Fibre Bundles......................................................146 
10.1 Invariant Polynomials and Characteristic Classes ........................................146

10.2 Chem Classes of Complex Vector Bundles................................................150

10.3 Pontrjagin Classes of Real Vector Bundles ..................................................154

10.4 Euler Classes of Real Oriented Even Dimensional Vector Bundles.................155

10.5 Stifel-Whitney Classes of Real Vector Bundles .............................................159

10.6 Chern-Simons Form - an Example of Secondary Classes..............................160

Part III. Index Theorems and 4-manifolds

Chapter 11. Index Theorems for Manifolds without Boundary ...............................162

11.1 Analytic Index and Elliptic Differential Operator .......................................162

11.2 Elliptic Complexes and Atiyah-Singer's Index Theorem .................................167

11.3 de Rham Complex and Gauss-Bonnet Theorem...........................................174

11.4 Index Theorems of Other Typical Elliptic Complexes ...................................181

Chapter 12. Essential Features of 4-Manifolds....................................................................182

12.1 Nontrivial Instanton Solutions $(* \mathrm{~F}=\mathrm{F})$ and Bianchi Identity on $\mathrm{S}^{4} \ldots \ldots \ldots \ldots \ldots \ldots . . . .182$

12.2 Dimension of the Moduli Space of the Self-dual Connection $A\left(\in \Lambda_{g}^{1}\right)$.........189

12.3 Topological Classification of Simply Connected 4-Manifolds.........................197

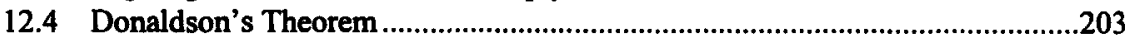

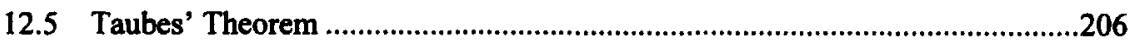

References................................................................................................................................207

Index 